\title{
Particle Dependence of Quenching Effect in an Optical-Fiber-Type Optically Stimulated Luminescence Dosimeter
}

\author{
Yuho Hirata, ${ }^{*}$ Kenichi Watanabe, Sachiko Yoshihashi, Akira Uritani, Yusuke Koba, ${ }^{1}$ \\ Naruhiro Matsufuji, ${ }^{1}$ Takayuki Yanagida, ${ }^{2}$ Toshiyuki Toshito, ${ }^{3}$ and Kentaro Fukuda ${ }^{4}$ \\ Graduate School of Engineering, Nagoya University, Furo, Chikusa, Nagoya, Aichi 464-8603, Japan \\ ${ }^{1}$ National Institute of Radiological Sciences, 4-9-1, Anagawa, Inage, Chiba, Chiba 263-8555, Japan \\ ${ }^{2}$ Nara Institute of Science and Technology, Takayama, Ikoma, Nara 630-0192, Japan \\ ${ }^{3}$ Nagoya Proton Therapy Center, 1-1-1, Hirate, Kita, Nagoya, Aichi 462-0057, Japan \\ ${ }^{4}$ Tokuyama Corporation, 1-1, Mikage, Shunan, Yamaguchi 745-8648, Japan
}

(Received April 7, 2017; accepted August 21, 2017)

Keywords: dosimeter, heavy-ion radiotherapy, optically stimulated luminescence, optical fiber probe, quenching phenomena

To monitor an actual dose in a patient's body during radiotherapy treatment, we have developed a small-size dosimeter of an optical fiber probe mounting optically stimulated luminescence (OSL) materials at the tip. The OSL materials show a quenching effect when they are irradiated by charged particles with high linear energy transfer (LET). We evaluate the particle dependence on the quenching effect in the small-size OSL dosimeter probe. $\mathrm{Eu}: \mathrm{BaFBr}$ and $\mathrm{Ce}: \mathrm{CaF}_{2}$ were adopted as OSL materials, and two types of small-size dosimeter were fabricated. We irradiated 290 $\mathrm{MeV} / \mathrm{u}$ carbon ions, $150 \mathrm{MeV} / \mathrm{u}$ helium ions, and $225 \mathrm{MeV}$ protons and measured the Bragg peak. The $\mathrm{Eu}: \mathrm{BaFBr}$ small-size dosimeter showed the quenching effect in these three types of particle irradiation. The $\mathrm{Ce}: \mathrm{CaF}_{2}$ small-size dosimeter showed the quenching effect only in carbon ion irradiation. The luminescence efficiencies of Eu:BaFBr were compared among these three charged particles. The quenching effect of $\mathrm{Eu}: \mathrm{BaFBr}$ is independent of the irradiated particles.

\section{Introduction}

In cancer treatments, radiation therapies attract attention as one of the low-burden treatments for patients. Particle therapies can form a three-dimensionally well-confined dose distribution, while X-ray radiotherapies deposit radiation energy not only into an affected area but also around the area. Bragg peaks of high-energy ions are sufficiently sharp to separately irradiate a tumor and normal organs. Among particle therapies, heavy-ion radiotherapy, in which high-energy carbon ions are usually used, has some excellent features. High-energy carbon ions generally have higher linear energy transfer (LET) than protons used in radiotherapies. Carbon ions, therefore, have higher relative biological effectiveness (RBE) than protons. The required irradiation treatment time can be short with a high-RBE radiation. ${ }^{(1-3)}$ Carbon ions are often used as prostatic cancer therapies because the prostate can selectively be irradiated without irradiating the rectum. The urethra is also a critical organ. Irradiation should be limited within an acceptable level in radiation 
therapies. The carbon ion beam has the possibility of irradiating the prostate without injuring the urethra.

Although the fine dose distribution can reduce the undesired irradiation of healthy organs or tissues, misalignment of an irradiation position may immediately cause significant accidental exposure and deficiency of the irradiation dose into a tumor. At present, the irradiation dose distribution is carefully planned and estimated using treatment planning software. In addition, the planned irradiation procedures are confirmed on the basis of phantom measurements as routine works. The dose on the surface of a patient's body is sometimes evaluated but the actual irradiation dose to an affected region during treatments is hardly monitored. In order to accurately evaluate the irradiation dose for extremely fine irradiation plans, direct measurements are desired for the irradiation dose in or around affected regions during treatments. For direct measurements, a dosimeter should be inserted into an affected region in a patient's body.

Optically stimulated luminescence (OSL) elements, which can accumulate radiation information as carriers are captured into trapping centers, are widely used as dosimeters. A stimulation light irradiation releases the captured carriers and the element emits OSL photons proportional to the irradiation dose. The OSL signal, therefore, can be a measure of the irradiation dose. ${ }^{(4)}$ The OSL element can accumulate the irradiation information and then release it instantly just after irradiating the stimulation light. This means that the OSL signals can be read out in the silent intervals of pulsed irradiations. The OSL measurement can avoid in-fiber light emission noise, which is generated during irradiation. The OSL dosimeter has an advantage especially as a small optical fiber probe compared with scintillator-type dosimeters. A small-size dosimeter consisting of an optical fiber and OSL was suggested. ${ }^{(5-7)}$

As OSL elements, we adopted $\mathrm{Eu}: \mathrm{BaFBr}$ and $\mathrm{Ce}_{\mathrm{CaF}} \mathrm{Ca}_{2} \mathrm{Eu}: \mathrm{BaFBr}$ is widely used in digital radiographic films called an imaging plate. $\mathrm{Ce}: \mathrm{CaF}_{2}$ is a relatively new OSL material. The Ce concentration in $\mathrm{Ce}: \mathrm{CaF}_{2}$ was $0.5 \%$. The OSL properties were reported in previous work. ${ }^{(8)}$ These OSL materials emit a relatively strong and fast OSL signal compared with other OSL materials. The dosimeter probe is fabricated with powdered OSL material adhered to a tip of the optical fiber with ultraviolet curing resin. The fabricated dosimeter is quite small, in which the size of the adhered OSL material is approximately $500 \mu \mathrm{m}$ in diameter and $100 \mu \mathrm{m}$ in thickness. Some basic performances of these small-size dosimeters were already evaluated. ${ }^{(9,10)}$

Since the LET of high-energy charged heavy particles, such as carbon ions, gradually increases with decreasing energy, a charged particle has LET variation along its track and has a quite high LET at the end of the track. The quenching phenomenon, which is the degradation of the luminescence efficiency in luminescence materials for high-LET particles, was reported by a number of researchers. ${ }^{(11-13)}$ This phenomenon was also observed in our small-size dosimeter, and the level of quenching was varied among the phosphors. ${ }^{(14,15)}$ The quenching effect is considered to be due to the temporal and local deficiencies of luminescence origins, such as trap centers in OSL materials. High-LET particles cause highly dense ionization and excitation. In the OSL process, excited electrons move in the conduction band and then fall into the trap centers in the OSL element. Stimulation light irradiation releases these trapped electrons and causes luminescence. Under high-density excitation along high-LET particle tracks, the trap centers are locally filled up with other excited electrons and a part of the electrons cannot fall into the trap centers. ${ }^{(16-18)}$ In this process, the range of secondary electrons is important. The range of secondary particles depends on materials. This is one of the possibilities for the change in OSL characteristics depending on irradiating particles. In this paper, we evaluate the particle dependence on the quenching effect in the small-size OSL dosimeters. 


\section{Materials and Methods}

We fabricated small-size optical-fiber-type dosimeter systems as shown in Fig. 1. Figure 1(a) shows the dosimeter system using Eu:BaFBr. This dosimeter system consists of quartz optical fibers (core diameter: $400 \mu \mathrm{m}$, numerical aperture: 0.22 ), a red laser diode as a stimulation light source $(630 \mathrm{~nm}$, BWT Beijing, K63S09F-0.40W), a photomultiplier tube (PMT, Hamamatsu, H6612), a timing control unit, a signal processing unit, and a personal computer to control the whole system and to acquire data. The dosimeter probe was connected to an optical fiber coupler and split into two ways. This optical fiber coupler divides photons into a ratio of 9 to 1 . A terminal of $10 \%$ branching was connected to the red laser diode stimulation light source. Another terminal of $90 \%$ branching was connected to the PMT. In order to avoid red laser reflection light, the bandpass filter (Thorlabs FB400-40) was mounted in front of the photocathode of the PMT. The center wavelength and transmission bandwidth of the band-pass filter are 400 and $50 \mathrm{~nm}$, respectively, which match the OSL wavelength of Eu:BaFBr. The laser diode operated in pulse mode with the duration of $50 \mathrm{~ms}$.

Figure 1(b) shows the dosimeter system using $\mathrm{Ce}: \mathrm{CaF}_{2}$. The basic configuration is the same as that of the Eu:BaFBr system. The laser diode (532 nm, Thorlabs, DJ532-40) was connected to a terminal of $90 \%$ branching of the optical fiber coupler. A PMT with a band-pass filter (275-375 $\mathrm{nm}$, Thorlabs, FGUV11) was connected to a terminal of $10 \%$ branching. The laser diode operated in $\mathrm{CW}$ mode but was pulsed into $400 \mathrm{~ms}$ duration by a mechanical shutter. The OSL signals were recorded through a digitizer into the control PC and analyzed. A dosimeter output was derived by integrating the luminescence signal over time.

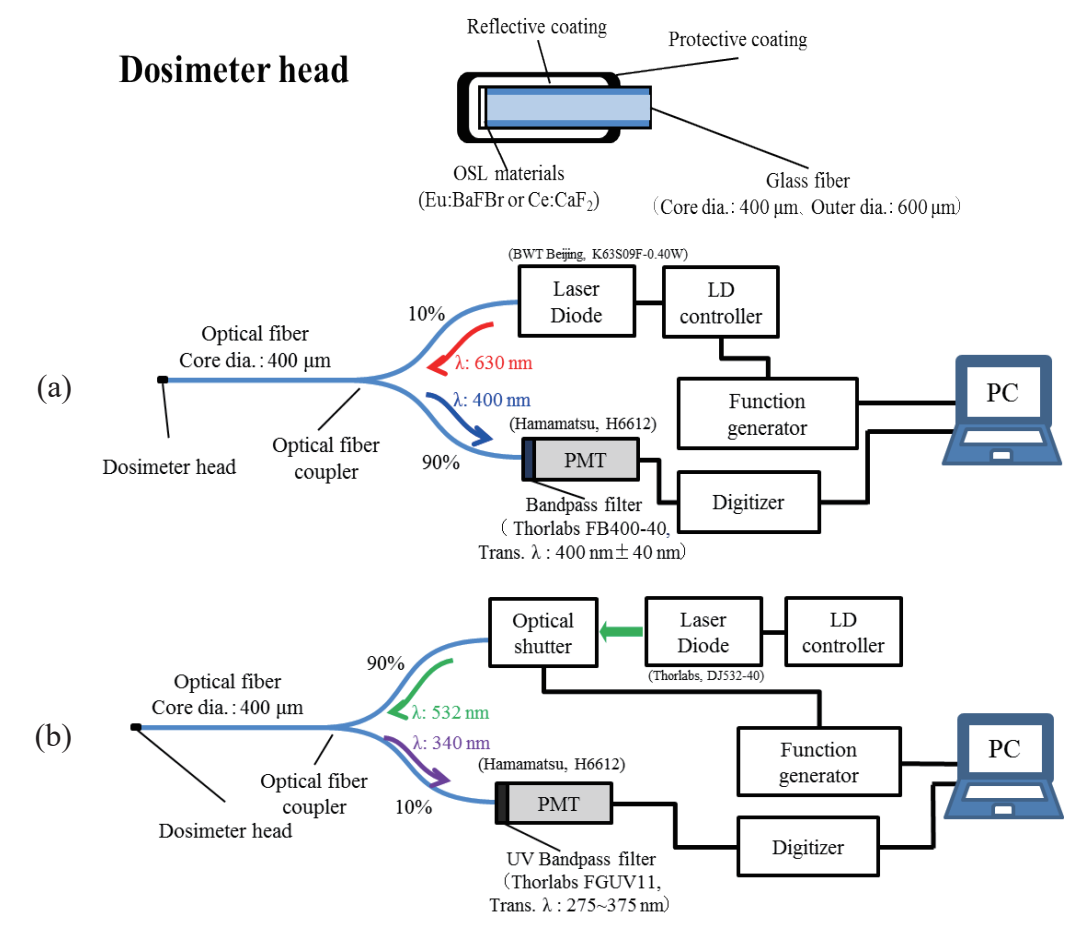

Fig. 1. (Color online) Schematic of optical-fiber-type small-size dosimeter system. This system consists of an OSL dosimeter probe, a laser diode, an optical-fiber-based light transmission system, a photomultiplier tube, and a data acquisition system. (a) The Eu:BaFBr small size dosimeter required the red laser diode and the bandpass filter of $420 \mathrm{~nm}$ transmission. (b) The $\mathrm{Ce}: \mathrm{CaF}_{2}$ small size dosimeter required the green laser diode and the bandpass filter of $340 \mathrm{~nm}$ transmission. 
The $290 \mathrm{MeV} / \mathrm{u}$ carbon ions and the $150 \mathrm{MeV} / \mathrm{u}$ helium ions were irradiated to the fabricated dosimeter at the Heavy Ion Medical Accelerator in Chiba (HIMAC, National Institute of Radiological Science in Japan). The amount of irradiated ions was monitored with a parallel-plate ion chamber located in the irradiation port and controlled using the output of the ion chamber. The ion beam had a round shape of $10 \mathrm{~cm}$ diameter.

The cycle period and ion beam pulse duration of HIMAC are 3.3 and $1.8 \mathrm{~s}$, respectively. The dosimeter system synchronized with ion beam pulses using the accelerator trigger signals. The OSL signal readout phase should be selected into intervals between the ion beam pulses, which are periods without ion beam irradiation, as shown in Fig. 2. The OSL signals were read out for every ion beam pulse.

Figure 3 shows the experimental arrangement for Bragg peak measurements. The fabricated small-size dosimeter was set at the center of the beam. A farmer-type ion chamber (PTW23343, Markus Ion Chamber) was also set just next to the fabricated dosimeter as a reference monitor. Water-equivalent acrylic phantoms with various thicknesses were placed in front of the dosimeters. The phantom total thickness was easily changed by changing the combination of the phantoms. We evaluated dosimeter responses at various depths from the phantom surface and obtained the energy deposition distribution as a function of depth.

The $225 \mathrm{MeV}$ protons were irradiated to the fabricated dosimeter at the Nagoya Proton Therapy Center. Figure 4 shows the experimental arrangement for Bragg peak measurements. An acrylic tank filled with water was located in the beam line. The fabricated dosimeter and the farmer-type ion chamber can be moved in a water tank with a linear stage. We measured the Bragg peak by moving the dosimeters in the axial direction of the proton beam. The amount of irradiated protons was controlled at $30 \mathrm{mGy}$ at the Bragg peak position in each readout. The proton irradiation experiment was conducted only for the $\mathrm{Eu}: \mathrm{BaFBr}$ small-size dosimeter.

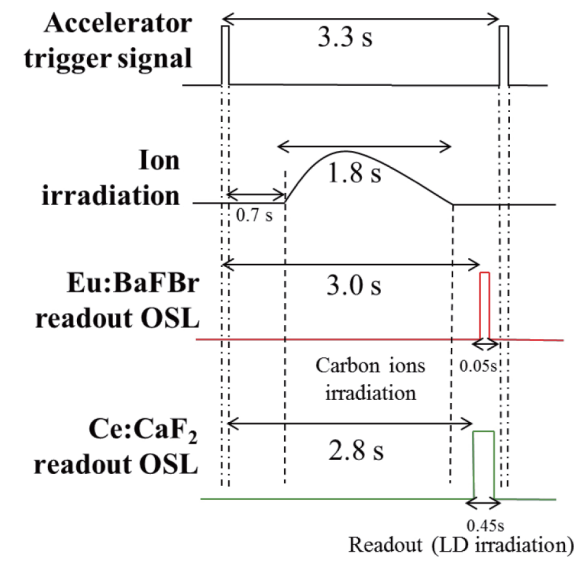

Fig. 2. (Color online) Timing chart of data acquisition of dosimeter systems and carbon or helium ion beam pulses of HIMAC. The readout timing was controlled by the delay generator. The signal readout time or stimulation laser pulse duration for the OSL dosimeters were set to be $50 \mathrm{~ms}$ for $\mathrm{Eu}: \mathrm{BaFBr}$ and $450 \mathrm{~ms}$ for $\mathrm{Ce}: \mathrm{CaF}_{2}$.

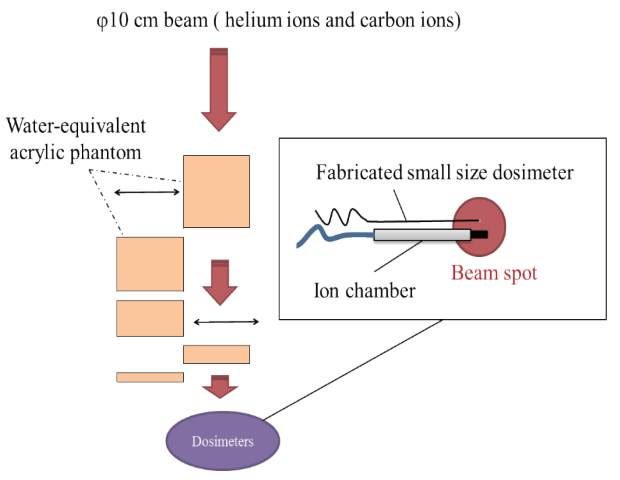

Fig. 3. (Color online) Experimental arrangement of Bragg peak measurements. The fabricated small size dosimeters and a reference ion chamber were set behind water-equivalent acrylic phantoms with various thicknesses and irradiated by 10 -cm-diameter ion beams, which include $290 \mathrm{MeV} / \mathrm{u}$ monoenergetic carbon ions and $150 \mathrm{MeV} / \mathrm{u}$ monoenergetic helium ions. 


\section{Results}

The small-size dosimeter responses to $290 \mathrm{MeV} / \mathrm{u}$ monoenergetic carbon ions were already evaluated. ${ }^{(14,15)}$ Figure 5 shows the phantom thickness dependence of signal intensities obtained from the fabricated small-size dosimeters. The measurements were carried out three times at each phantom thickness. The standard deviations were evaluated from three measurements. The actual dose distribution measured with the reference ion chamber is also plotted. The signal intensities are normalized at zero thickness corresponding to a patient's surface. The fabricated dosimeters showed the quenching effect near the Bragg peak.

Figure 6 shows the phantom thickness dependence of signal intensities obtained from the fabricated small-size dosimeters and the ion chamber when irradiating $150 \mathrm{MeV} / \mathrm{u}$ monoenergetic helium ions. The signal intensities are normalized at zero thickness. The dose distribution

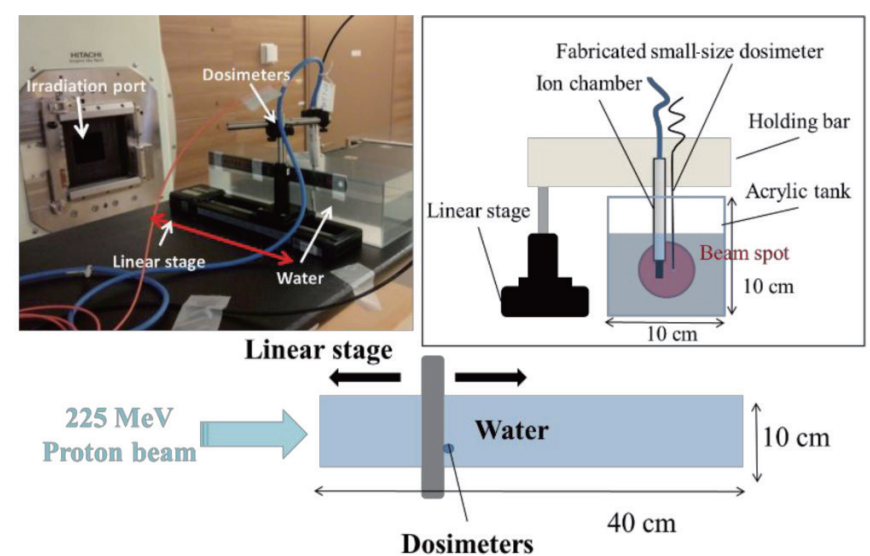

Fig. 4. (Color online) Experimental arrangement of Bragg peak measurements for $225 \mathrm{MeV}$ protons. The fabricated small-size dosimeters and a reference ion chamber were held by a linear stage and set in water. The linear stage moved to the axial direction of the proton beam.

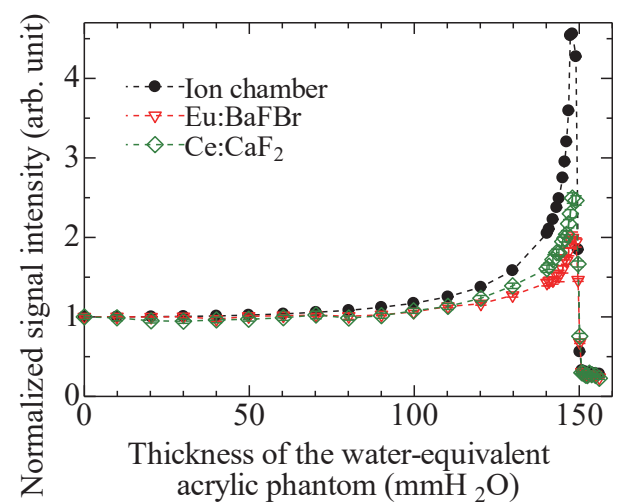

Fig. 5. (Color online) Phantom thickness dependence of signal intensities obtained from the fabricated small-size dosimeters and the ion chamber when the dosimeters were irradiated with $290 \mathrm{MeV} / \mathrm{u}$ monoenergetic carbon ions.

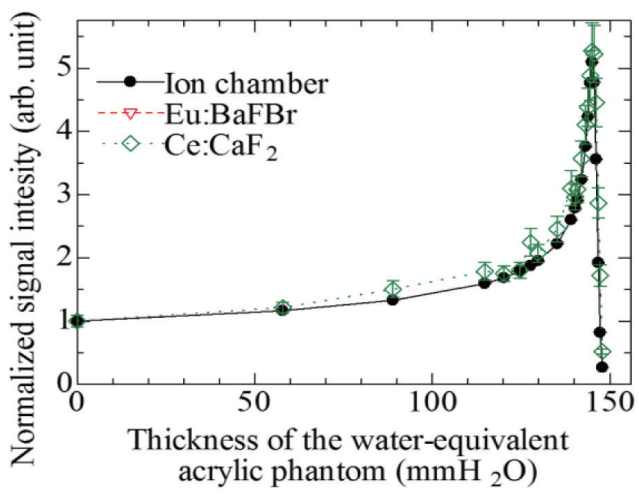

Fig. 6. (Color online) Phantom thickness dependence of signal intensities obtained from the fabricated small-size dosimeters and the ion chamber when the dosimeters were irradiated with $150 \mathrm{MeV} / \mathrm{u}$ monoenergetic helium ions. 
obtained from the fabricated dosimeters has a Bragg peak at $145 \mathrm{~mm}$ thickness. At the same position, the reference ion chamber also shows the peak. The Eu:BaFBr dosimeter showed a lower Bragg peak than the ion chamber owing to the quenching effect. On the other hand, the Ce:CaF dosimeter showed no quenching effect even near the Bragg peak.

Figure 7 shows the depth dependence of signal intensities obtained from the $\mathrm{Eu}: \mathrm{BaFBr}$ dosimeter and the ion chamber irradiated with $225 \mathrm{MeV}$ monoenergetic protons. The depth of each dosimeter was adjusted at the Bragg peak position. The signal intensities of the Eu:BaFBr dosimeter were also quenched at the Bragg peak.

\section{Discussion}

The luminescence efficiency is defined as the ratio of the signal intensities of the fabricated dosimeters and the ion chamber. The luminescence efficiencies of $\mathrm{Eu}: \mathrm{BaFBr}$ at the Bragg peak with carbon ions, helium ions, and protons were $0.41,0.50$, and 0.89 , respectively. The quenching effect was stronger with heavier particles.

The averaged LET at each phantom thickness or depth in the water was calculated with the Monte Carlo calculation Particle and Heavy Ion Transport code System (PHITS). ${ }^{(19)}$ In this calculation, a cylindrical water phantom of $20 \mathrm{~cm}$ diameter and $20 \mathrm{~cm}$ thickness was irradiated by a 10 -cm-diameter beam. The averaged LET was calculated within $2 \mathrm{~cm}$ diameter from the center of the beam. The calculation step in the depth direction was $200 \mu \mathrm{m}$.

Figure 8 shows the relationship between the normalized luminescence efficiencies and the averaged LET when the dosimeters were irradiated with $290 \mathrm{MeV} / \mathrm{u}$ carbon ions, which were already reported. ${ }^{(14,15)}$ These efficiencies were normalized at zero thickness, where the LET was $14.41 \mathrm{keV} / \mu \mathrm{m}$. The luminescence efficiencies of $\mathrm{Eu}: \mathrm{BaFBr}$ and $\mathrm{Ce}: \mathrm{CaF}_{2}$ decreased monotonically as the averaged LET increases.

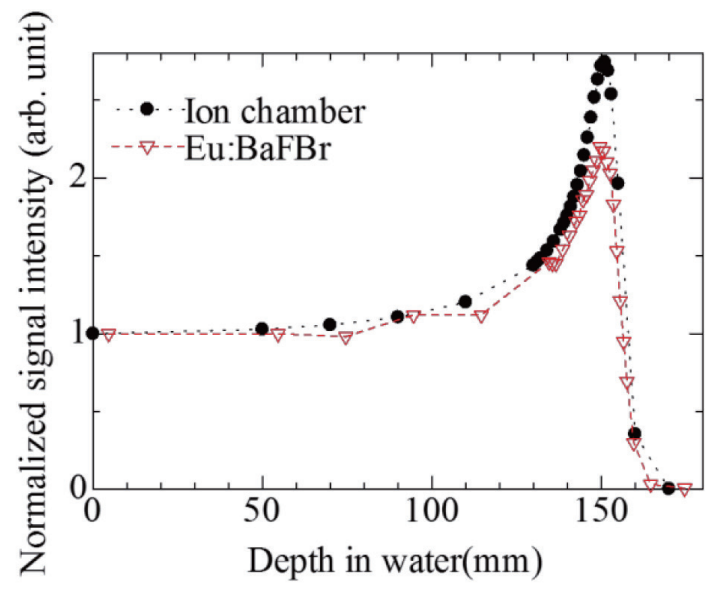

Fig. 7. (Color online) Water depth dependence of signal intensities obtained from the fabricated small-size dosimeters and the ion chamber when the dosimeters were irradiated with $225 \mathrm{MeV}$ monoenergetic protons.

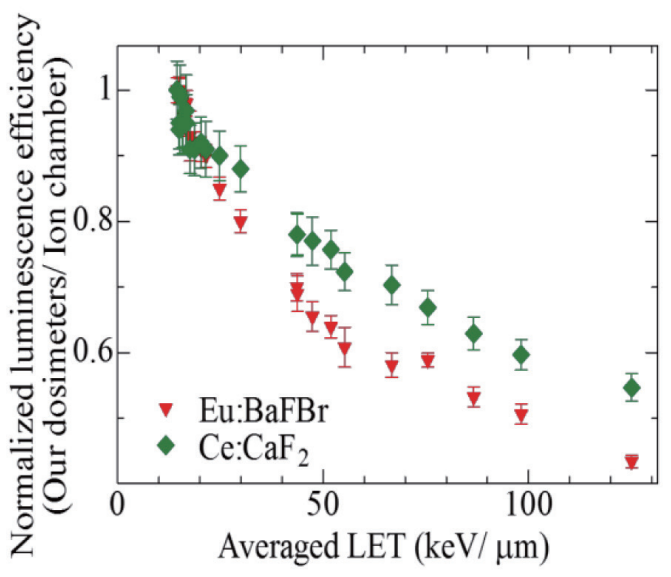

Fig. 8. (Color online) Averaged LET dependence of the luminescence efficiencies of the $\mathrm{Eu}: \mathrm{BaFBr}$ and $\mathrm{Ce}: \mathrm{CaF}_{2}$ small-size dosimeters when the dosimeters were irradiated with $290 \mathrm{MeV} / \mathrm{u}$ monoenergetic carbon ions. 
Figure 9 shows the relationship between the normalized luminescence efficiencies and the averaged LET when the dosimeters were irradiated with $150 \mathrm{MeV} / \mathrm{u}$ helium ions. These efficiencies were also normalized at zero thickness, where the LET was $4.64 \mathrm{keV} / \mu \mathrm{m}$. The luminescence efficiency of Eu:BaFBr was decreased with the averaged LET. The luminescence efficiency of $\mathrm{Ce}: \mathrm{CaF}_{2}$ had no dependence on the increase in the averaged LET.

Figure 10 shows the relationship between the normalized luminescence efficiencies and the averaged LET when the dosimeters were irradiated with $225 \mathrm{MeV} / \mathrm{u}$ protons. These efficiencies were also normalized at zero depth, where the LET was $0.95 \mathrm{keV} / \mu \mathrm{m}$. The luminescence efficiency of the $\mathrm{Eu}: \mathrm{BaFBr}$ dosimeter monotonically decreases with increasing the averaged LET.

To evaluate the particle dependence on the luminescence efficiency, we compare the luminescence efficiencies of Eu:BaFBr for each particle as shown in Fig. 11. The luminescence

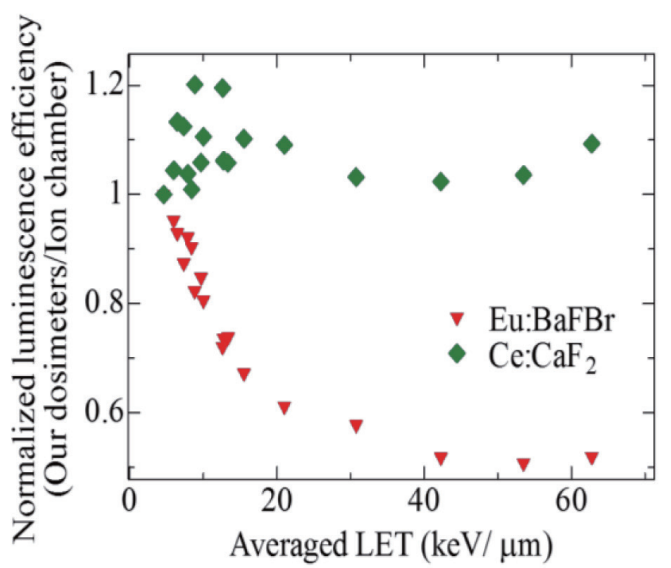

Fig. 9. (Color online) Averaged LET dependence of the luminescence efficiencies of the $\mathrm{Eu}: \mathrm{BaFBr}$ and $\mathrm{Ce}: \mathrm{CaF}_{2}$ small-size dosimeters when the dosimeters were irradiated with $150 \mathrm{MeV} / \mathrm{u}$ monoenergetic helium ions.

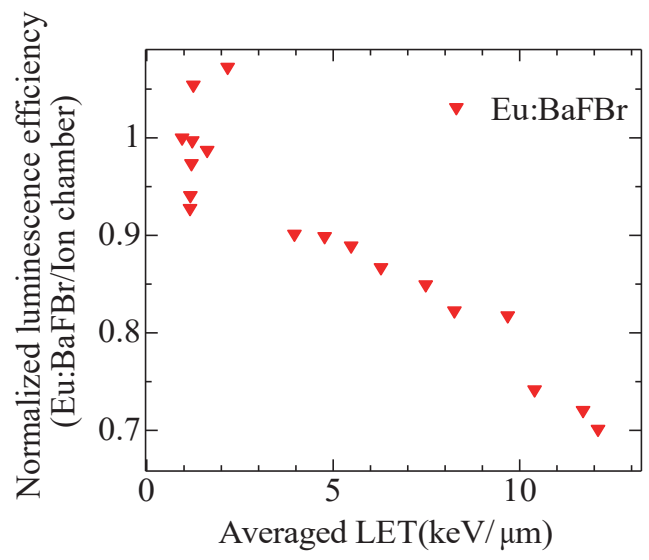

Fig. 10. (Color online) Averaged LET dependence of the luminescence efficiencies of the Eu:BaFBr smallsize dosimeter when the dosimeters were irradiated with $225 \mathrm{MeV}$ monoenergetic protons.

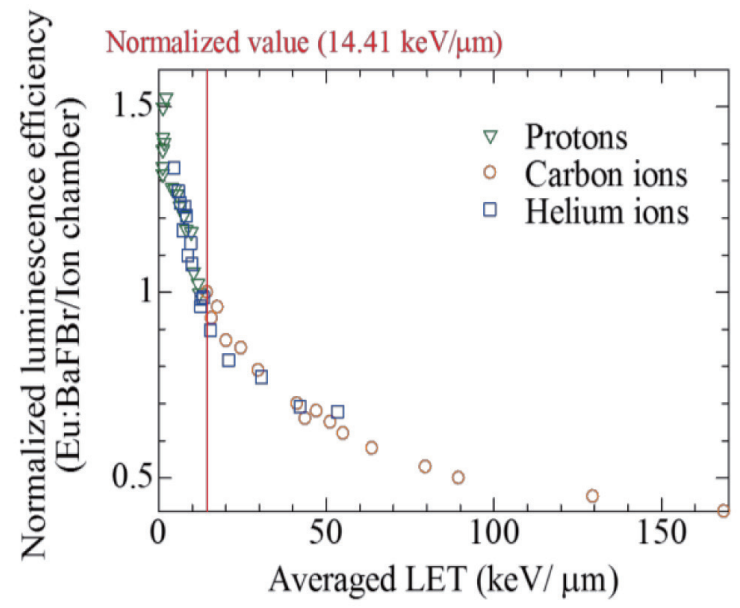

Fig. 11. (Color online) Comparison of the luminescence efficiencies of Eu:BaFBr small-size dosimeter with irradiated particles. The luminescence efficiencies when helium ions and protons were irradiated were normalized at $14.41 \mathrm{keV} / \mu \mathrm{m}$, which was the averaged LET at the $0 \mathrm{~mm}$ depth of carbon ion irradiation. 
efficiency was normalized at the averaged LET of $14.41 \mathrm{keV} / \mu \mathrm{m}$, which was the averaged LET of carbon ions at zero thickness. We confirmed no difference in the behavior of the luminescence efficiencies among the three types of particle. The Eu:BaFBr small-size dosimeter might be corrected with the LET information even under irradiation with various types of particle.

The luminescence efficiency of $\mathrm{Eu}: \mathrm{BaFBr}$ has no dependence on the types of irradiated particle, whereas that of $\mathrm{Ce}: \mathrm{CaF}_{2}$ significantly changed between helium ions and carbon ions. The investigation of the irradiated particle dependence of the mechanism of the quenching effect in OSL materials will be a future work.

\section{Conclusions}

To evaluate the irradiated particle dependence on the quenching effect in OSL materials, we conducted irradiation experiments using carbon ions, helium ions, and protons. The irradiations of helium ions and carbon ions were conducted at HIMAC with the Eu:BaFBr and Ce:CaF 2 small-size dosimeter. The proton irradiation was conducted at the Nagoya Proton Therapy Center with the $\mathrm{Eu}: \mathrm{BaFBr}$ one.

The Eu:BaFBr small-size dosimeter showed the quenching effect in carbon ion, helium ion, and proton irradiations. The Ce: $\mathrm{CaF}_{2}$ small-size dosimeter also showed the quenching effect in carbon ions, whereas it shows no quenching effect under the helium ion irradiation. The averaged LET dependence of the luminescence efficiencies of the Eu:BaFBr is independent of the irradiated particles. Systematic investigations on the luminescence behaviors should be performed as future works.

\section{Acknowledgement}

This work was supported by JSPS KAKENHI Grant Number JP17J05849.

\section{References}

1 H. Tsujii, J. Mizoe, T. Kamada, M. Baba, H. Tsuji, H. Kato, S. Kato, S. Yamada, S. Yasuda, T. Ohno, T. Yanagi, R. Imai, K. Kagei, H. Kato, R. Hara, A. Hasegawa, M. Nakajima, N. Sugane, N. Tamaki, R. Takagi, S. Kandatsu, K. Yoshikawa, R. Kishimoto, and T. Miyamoto: J. Radiat. Res. 48 (2007) A1.

2 J. J. Wilkens and U. Oelfke: Int. J. Radiat. Oncol. 70 (2008) 262.

3 T. Elsässer, W. K. Weyrather, T. Friedrich, M. Durante, G. Iancu, M. Krämer, G. Kragl, S. Brons, M. Winter, K.-J. Weber, and M. Scholz: Int. J. Radiat. Oncol. 78 (2010) 1177.

4 Y. Iwabuchi, N. Mori, and K. Takahashi: Fujifilm Res. Dev. 44 (1999) 37.

5 D. M. Klein and S. W. S. Mckeever: Radiat. Meas. 43 (2008) 883.

6 D. Klein, D. W. Peakheart, and S. W. S. Mckeever: Radiat. Meas. 45 (2010) 663.

7 R. Gaza and S. W. S. Mckeever: Radiat. Prot. Dosim. 120 (2006) 14.

8 T. Yanagida, Y. Fujimoto, K. Watanabe, K. Fukuda, N. Kawaguchi, Y. Miyamoto, and H. Nanto: Radiat. Meas. 71 (2014) 162.

9 K. Watanabe, A. Yamazaki, K. Nakahashi, H. Miyamae, A. Uritani, and E. Ariga: Radiat. Meas. 55 (2013) 64.

10 Y. Hirata, K. Watanabe, A. Yamazaki, A. Uritani, Y. Koba, N. Matsufuji, T. Yanagida, and K. Fukuda: Ionizing Radiat. 41 (2016) 157.

11 A. Nohtomi, T. Terunuma, R. Kohno, Y. Takada, Y. Hayakawa, A. Maruhashi, and T. Sakae: Nucl. Instrum. Methods Phys. Res., Sect. A 424 (1999) 569.

12 L. Torrisi: Nucl. Instrum. Methods Phys. Res., Sect. B 170 (2000) 523.

13 L. Archambault, J. C. Polf, L. Beaulieu, and S. Beddar: Phys. Med. Biol. 53 (2008) 1865.

14 Y. Hirata, K. Watanabe, A. Uritani, A. Yamazaki, Y. Koba, and N. Matsufuji: J. Radiat. Prot. Res. 41 (2016) 228. 
15 Y. Hirata, K. Watanabe, A. Uritani, A. Yamazaki, Y. Koba, N. Matsufuji, T. Yanagida, and K. Fukuda: Radiat. Meas. (in press).

16 M. Thoms, H. von Seggern, and A. Winnacker: Phys. Rev. B 44 (1991) 9240.

17 P. Olko: Radiat. Meas. 41 (2006) 57.

18 A. Menchaca-Rocha: Nucl. Instrum. Methods Phys. Res., Sect. A 602 (2009) 421.

19 T. Sato, K. Niita, N. Matsuda, S. Hashimoto, Y. Iwamoto, S. Noda, T. Ogawa, H. Iwase, H. Nakashima, T. Fukahori, K. Okumura, T. Kai, S. Chiba, T. Furuta, and L. Sihver: J. Nucl. Sci. Technol. 529 (2013) 913.

\section{About the Authors}

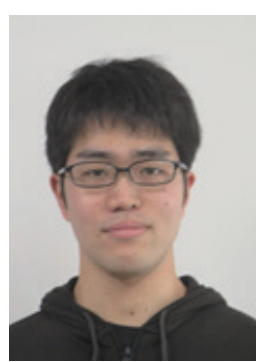

Yuho Hirata received his B.S. and M.S. degrees from Nagoya University, Japan, in 2015 and 2017, respectively. Since 2017, he has been a Ph.D. student at Nagoya University, Japan. His research interests are in radiation measurements and dosimetry.

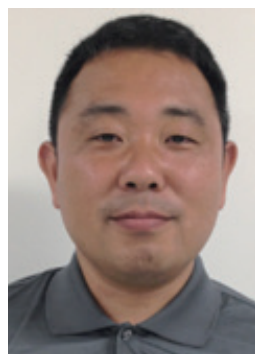

Kenichi Watanabe received his M.S. and Ph.D. degrees from Nagoya University, Japan, in 1998 and 2003, respectively. From 2000 to 2007, he was a research assistant at Nagoya University, Japan. Since 2007, he has been an associate professor at Nagoya University. His research interests are in radiation detectors.

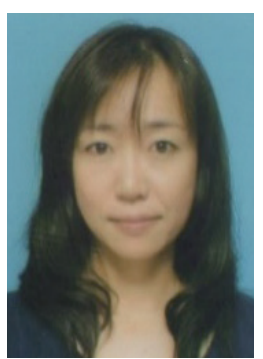

Sachiko Yoshihashi received her B.S. degree from Gifu University, Japan, in 1996 and her M.S. and Ph.D. degrees from Osaka University, Japan, in 1998 and 2001, respectively. She was a specially appointed research fellow and an assistant professor at the Graduate School of Engineering, Osaka University, from 2001 to 2014. On 2015, she was employed as an associate professor at Fukui University of Technology. Since 2016, she has been an associate professor at Nagoya University. Her current research interest is technical development for radiation medicine.

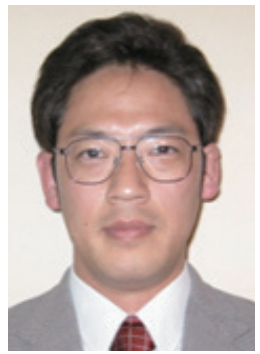

Akira Uritani received his B.S., M.S., and Ph.D. degrees from Nagoya University, Japan, in 1984, 1986, and 1990, respectively. From 1990 to 1998, he was an assistant professor at Nagoya University, Japan. Since 2005, he has been a professor at Nagoya University. His research interests are development of radiation detectors, thermonuclear fusion, and boron neutron capture therapy. 


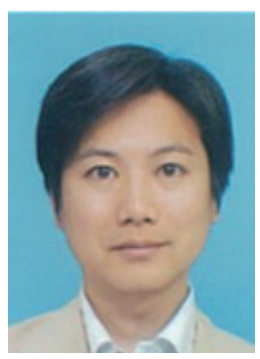

Yusuke Koba received his B.S., M.S., and Ph.D. degrees from Kyushu University, Japan, in 2005, 2007, and 2010, respectively. Since 2010, he has been a researcher at the National Institute of Radiological Sciences. His research interests are in medical physics, dosimetry, and detectors.

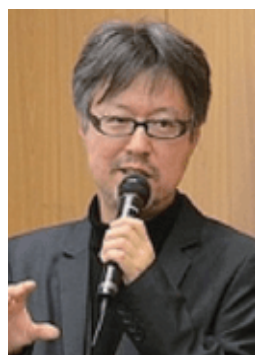

Naruhiro Matsufuji received his B.S., M.S., and Ph.D. degrees from Kyushu University, Japan, in 1992, 1994, and 2000, respectively. Since 1994, he has been working at the National Institute of Radiological Sciences, Japan, and as a Team Leader since 2006. Since 2010, he has been an adjunct professor at Tokyo Institute of Technology. His research interests are in medical and radiation physics, as well as radiation detection.

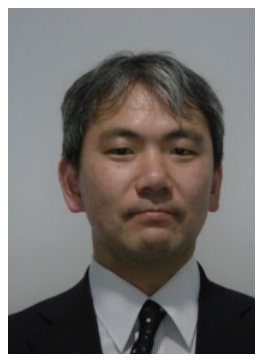

Takayuki Yanagida received his B.S., M.S., and Ph.D. degrees from The University of Tokyo, Japan, in 2002, 2004, and 2007, respectively. From 2007 to 2011, he was an assistant and associate professor at Tohoku University, Japan. From 2012, he was an associate professor at Kyushu Institute of Technology. Since 2015, he is a professor at Nara Institute of Science and Technology. His research interests are in scintillators, storage phosphors, and radiation detectors.

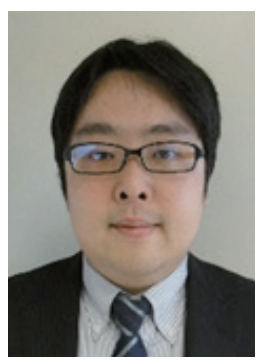

Toshiyuki Toshito received his B.S., M.S. and Ph.D. degrees from Nagoya University, Japan, in 1993, 1995, and 1999, respectively. Since 2012, he has been a medical physicist at Nagoya Proton Therapy Center. His research interests are in proton therapy physics.

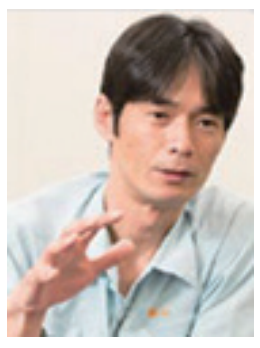

Kentaro Fukuda received his B.S. degree from Kyushu University, Japan, in 1998, M.S. degree from Nara Institute of Science and Technology, Japan, in 2000, and Ph.D. degree from Tohoku University, Japan, in 2011. Since 2000, he has been a research chemist at Tokuyama Corporation. His research interests are in inorganic materials for optics, phosphors, and scintillators. 